\title{
Broadband terahertz wave generation from an epsilon-near-zero material
}

\author{
Wenhe Jia', Meng Liu², Yongchang Lu², Xi Feng ${ }^{2}$, Qingwei Wang ${ }^{2}$, Xueqian Zhang ${ }^{2}$, Yibo Ni ${ }^{1}$ Futai Hu', Mali Gong ${ }^{1}$, \\ Xinlong $\mathrm{Xu}^{3}$, Yuanyuan Huang ${ }^{3}$, Weili Zhang $\mathbb{1}^{4}$, Yuanmu Yang ${ }^{1}$ and Jiaguang Han $\mathbb{\circledR}^{2}$
}

\begin{abstract}
Broadband light sources emitting in the terahertz spectral range are highly desired for applications such as noninvasive imaging and spectroscopy. Conventionally, THz pulses are generated by optical rectification in bulk nonlinear crystals with millimetre thickness, with the bandwidth limited by the phase-matching condition. Here we demonstrate broadband $\mathrm{THz}$ emission via surface optical rectification from a simple, commercially available $19 \mathrm{~nm}$ thick indium tin oxide (ITO) thin film. We show an enhancement of the generated THz signal when the pump laser is tuned around the epsilon-near-zero (ENZ) region of ITO due to the pump laser field enhancement associated with the ENZ effect. The bandwidth of the THz signal generated from the ITO film can be over $3 \mathrm{THz}$, unrestricted by the phasematching condition. This work offers a new possibility for broadband $\mathrm{THz}$ generation in a subwavelength thin film made of an ENZ material, with emerging physics not found in existing nonlinear crystals.
\end{abstract}

\section{Introduction}

Terahertz (THz) radiation spanning from 0.1 to $10 \mathrm{THz}$ falls between the microwave and infrared spectral ranges ${ }^{1}$. In recent years, $\mathrm{THz}$ technology applications have been rapidly expanding ${ }^{2,3}$ in areas including nondestructive material evaluation ${ }^{4,5}$, imaging ${ }^{6,7}$, sensing ${ }^{8,9}$, and wireless communication $^{10,11}$. $\mathrm{THz}$ radiation can be generated through the nonlinear downconversion of optical signals or through the nonlinear upconversion of microwave signals, among which the nonlinear optical rectification method is particularly popular for the generation of broadband $\mathrm{THz}$ pulses for spectroscopy-related applications. Despite the recent discoveries of broadband $\mathrm{THz}$ generation in air plasma ${ }^{12,13}$ and liquids ${ }^{14}$, THz emission is more routinely generated by pumping solid-state noncentrosymmetric nonlinear crystals, such as $\mathrm{ZnTe}^{15}$,

Correspondence: Yuanmu Yang (ymyang@tsinghua.edu.cn) or

Jiaguang Han (jiaghan@tju.edu.cn)

'State Key Laboratory of Precision Measurement Technology and Instruments, Department of Precision Instrument, Tsinghua University, Beijing 100084, China ${ }^{2}$ Center for THz Waves and College of Precision Instrument and

Optoelectronics Engineering, Tianjin University, Tianjin 300072, China

Full list of author information is available at the end of the article

These authors contributed equally: Wenhe Jia, Meng Liu
$\mathrm{GaP}^{16}$, and $\mathrm{LiNbO}_{3}{ }^{17}$, with a femtosecond laser typically operating in the near-infrared range. However, the intensity and bandwidth of the generated $\mathrm{THz}$ signal, as well as the pump wavelength, are often limited by the phase-matching condition in the bulk nonlinear crystal. This problem has recently stimulated growing interest in developing ultrathin $\mathrm{THz}$ emitters with thicknesses down to even a few atomic layers. In particular, optical metamaterials composed of split-ring resonators with magnetic dipole resonances have been identified as excellent nonlinear $\mathrm{THz}$ sources, with a spectral bandwidth unrestricted by the phase-matching condition, as well as the material absorption in the Reststrahlen region of conventional nonlinear crystals $^{18,19}$. However, the highly sophisticated nanofabrication process and the low laser damage threshold have largely hindered their wide adoption. Nanoscale $\mathrm{THz}$ emitters have also been realized with monolayer graphene ${ }^{20}$ and tungsten disulfide ${ }^{21}$, although with limited efficiency. Furthermore, surface $\mathrm{THz}$ emission from semiconductors such as InAs, InSb, and GaAs has been investigated ${ }^{22,23}$, although efficient $\mathrm{THz}$ emission has only been shown in the reflection configuration. 
More recently, materials that exhibit a vanishing real part of their permittivity in certain spectral ranges ${ }^{24,25}$, commonly known as epsilon-near-zero (ENZ) materials, have drawn much attention in the field of nonlinear optics $^{26-29}$ for applications such as second- and thirdharmonic generation ${ }^{30-32}$, all-optical switching ${ }^{33,34}$, and tuneable absorption ${ }^{35}$. The enhancement of nonlinear optical responses in materials within a subwavelength thickness, induced by the ENZ effect, can be partially explained by the amplification of the pump laser field due to the continuity of the normal displacement field at the boundary of the ENZ material and the background medium, following the formula $\varepsilon_{\mathrm{ENZ}} \cdot E_{\mathrm{ENZ}}=\varepsilon_{0} \cdot E_{0}$, where $\varepsilon_{\mathrm{ENZ}}$ and $\varepsilon_{0}$ are the permittivities of the ENZ material and background medium, respectively, and $E_{\mathrm{ENZ}}$ and $E_{0}$ are the normal components of the electric fields at the material boundary. When $\varepsilon_{\mathrm{ENZ}}$ approaches zero, the electric field in the ENZ material can be significantly enhanced. The enhanced nonlinearity in the ENZ material can be alternatively explained by the small group velocity, also known as the slow-light effect ${ }^{36}$, in the Ferrell-Berreman mode or the ENZ mode supported in the ENZ thin film.

ENZ effects occur in a wide range of materials, including transparent conducting oxides (TCOs) ${ }^{27}$, doped semiconductors ${ }^{37}$, and polar dielectrics ${ }^{38}$. Indium tin oxide (ITO) is one of the most widely available ENZ materials. It has been used as transparent electrodes in solar cells and consumer electronics. The dispersion of the permittivity of ITO follows the Drude-Lorentz formula $\varepsilon=\varepsilon_{\infty}+\omega_{\mathrm{p}, \mathrm{b}}^{2} /\left(\omega_{0, \mathrm{~b}}^{2}-\omega^{2}-i \gamma_{\mathrm{b}} \omega\right)-\omega_{\mathrm{p}}^{2} / \omega\left(\omega+i \gamma_{\mathrm{f}}\right)$, where $\varepsilon_{\infty}$ is the high-frequency permittivity, $\omega_{\mathrm{p}, \mathrm{b}}, \omega_{0, \mathrm{~b}}$, and $\gamma_{\mathrm{b}}$ are the plasma frequency, resonance frequency, and plasma damping rate of bound electrons, respectively, and $\omega_{\mathrm{p}}$ and $\gamma_{\mathrm{f}}$ are the plasma frequency and plasma damping rate of free electrons, respectively ${ }^{39}$. The plasma frequency of ITO further follows the formula $\omega_{\mathrm{p}}=\sqrt{N_{\mathrm{e}} e^{2} / m_{\mathrm{eff}} \varepsilon_{0}}$, where $N_{\mathrm{e}}$ is the carrier density, $\varepsilon_{0}$ is the permittivity in vacuum, and $m_{\text {eff }}$ is the ensembleaveraged effective electron mass. The carrier density of ITO is typically in the range of $10^{20}-10^{21} \mathrm{~cm}^{-3}$, leading to an ENZ wavelength $\lambda_{\text {ENZ }}$ typically in the near-infrared region.

In this work, we show $\mathrm{THz}$ generation from commercially available ITO glass by leveraging the ENZ effect. We observe THz emission from a $19 \mathrm{~nm}$-thick ITO film when pumped around its ENZ wavelength. The bandwidth of the generated $\mathrm{THz}$ pulse is $\sim 3 \mathrm{THz}$, limited only by the bandwidth of the pump laser and the detection crystal. We measure the pump wavelength, power, and polarization dependence to confirm that the $\mathrm{THz}$ emission originates from surface optical rectification in the ITO film and is enhanced by the ENZ effect. Moreover, we observe that the most efficient $\mathrm{THz}$ generation initially occurs at a pump wavelength slightly blueshifted from the static bulk $\lambda_{\mathrm{ENZ}}$ of ITO, which redshifts as the pump fluence increases, which may be associated with the nonlocal effect as well as the unique hot-electron dynamics in the ITO film.

\section{Results \\ Sample characteristics and measurement setup}

A schematic of the commercially available ITO on a glass substrate (PGO GmbH) is depicted in Fig. 1a. The film is found to be amorphous from the X-ray diffraction measurement, with a root mean square roughness of $0.35 \mathrm{~nm}$ determined from the atomic force microscopy measurement (see Supplementary Fig. 1 and Supplementary Note 1 for details). The real and imaginary parts of the relative permittivity and the thickness of the ITO film are measured through spectroscopic ellipsometry, as shown in Fig. 1b (see Supplementary Note 2 for details).
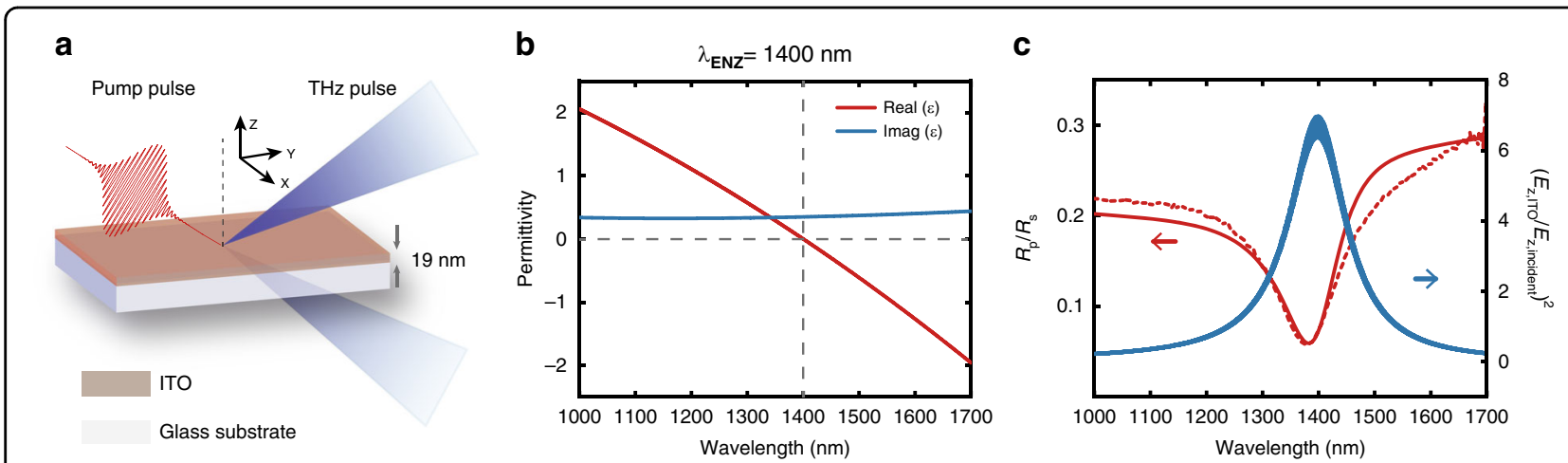

Fig. 1 Static optical response of the ITO film. a Schematic of the ENZ sample composed of a 19-nm-thick ITO layer and a $1.1 \mathrm{~mm}$-thick glass substrate. $\mathbf{b}$ Real and imaginary parts of the permittivity of the ITO film as a function of wavelength measured via spectroscopic ellipsometry. The ENZ wavelength is marked with a grey dashed line. $\mathbf{c} R_{\mathrm{p}} / R_{\mathrm{s}}$ and $\left(E_{\mathrm{Z}, \mathrm{T}} \mathrm{O} / E_{0, \text { incident }}\right)^{2}$ measured (dashed line) and calculated by the transfer matrix method (solid lines) as a function of the wavelength with an incident angle of $40^{\circ}$, where $R_{\mathrm{p}}$ and $R_{\mathrm{s}}$ are the reflectance of $p$-polarized and $s$-polarized light, respectively, and $E_{z, I T O}$ and $E_{z, \text { incident }}$ are the normal components of the electric field in the ITO film and the incident electric field, respectively 
The real part of its permittivity crosses zero at a wavelength of $1400 \mathrm{~nm}$, with an imaginary part of 0.35 . The $p$ polarized linear reflectance spectrum of the sample is measured at an incident angle of $40^{\circ}$, showing a resonance dip near its $\lambda_{\mathrm{ENZ}}$ as a result of the excitation of the Ferrell-Berreman mode ${ }^{25,40}$, in close agreement with the calculation, as illustrated in Fig. 1c. The corresponding electric field enhancement in ITO near its $\lambda_{\mathrm{ENZ}}$ is also calculated using the transfer matrix method.

The key elements of the $\mathrm{THz}$ time-domain emission spectroscopy system in both the transmission and reflection configurations are schematically shown in Fig. 2a. We use the output of an optical parametric amplifier (OPA) to excite the ITO sample with a wavelength tuneable from 1100 to $1600 \mathrm{~nm}$ in both the transmission and reflection configurations (see 'Materials and Methods' for details). Figure 2b, c illustrate the measured $\mathrm{THz}$ time-domain signals. We observe $\mathrm{THz}$ emission from the ITO sample in both the transmission and reflection configurations. In contrast, no $\mathrm{THz}$ emission is observed from a bare glass substrate under identical optical excitation conditions. We attribute the origin of the $\mathrm{THz}$ generation from the ultrathin ITO film to the surface second-order optical nonlinearity. Similar observations on the surface second-order optical nonlinearity of ITO have been made in previous literature studying the second-harmonic generation of ITO films around their ENZ wavelength ${ }^{39,41,42}$.

\section{a}
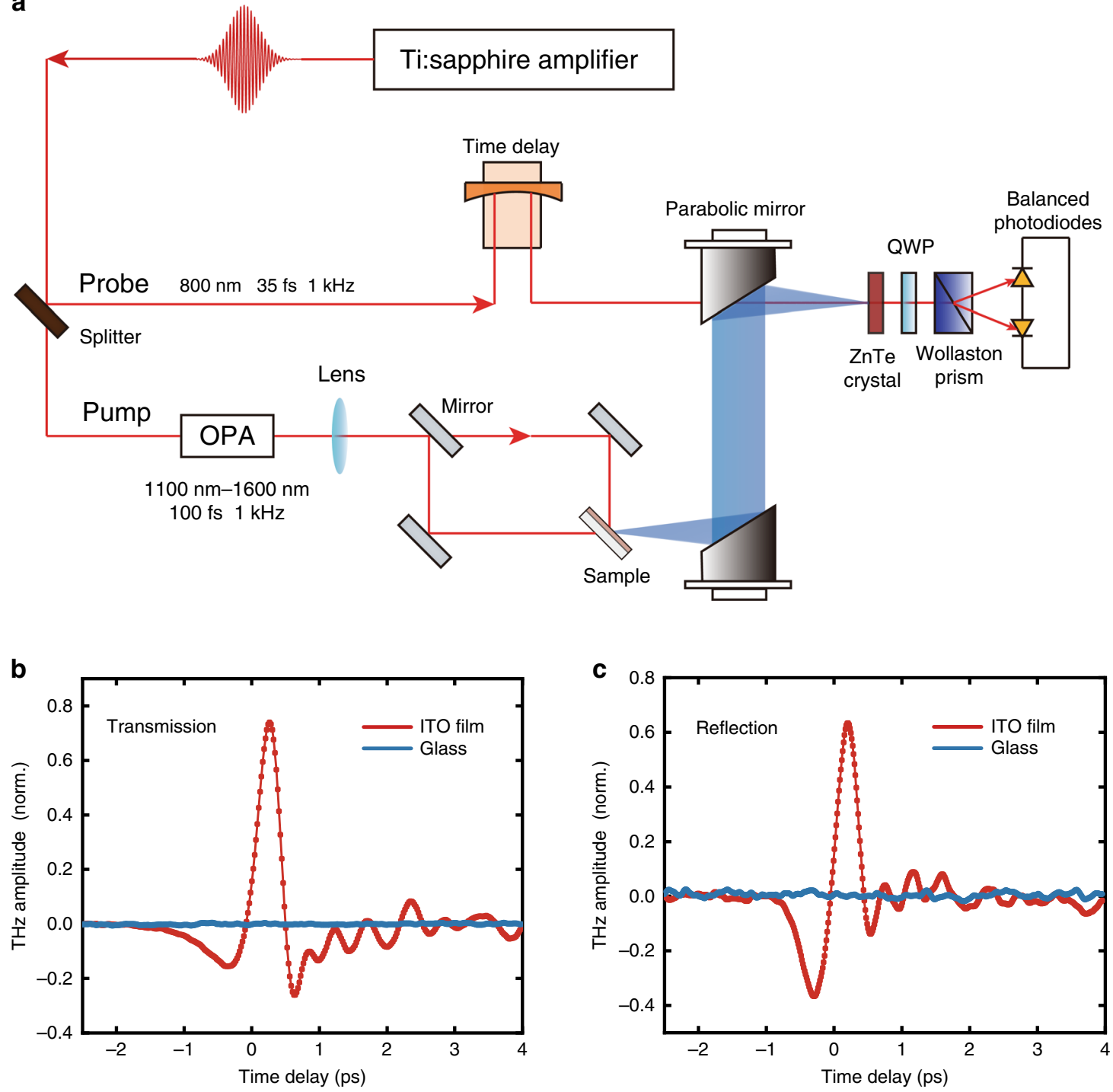

Fig. 2 Experimental setup and time-domain THz signals from the ITO film. a Schematics of the THz generation and detection setup in both the transmission and reflection configurations. b, c Time-domain THz signal measured by pumping the ITO film (red) and $0.5 \mathrm{~mm}$-thick bare glass substrate (blue) with pump wavelengths of 1350 and $1400 \mathrm{~nm}$ in the transmission and reflection configurations, respectively 


\section{Pump wavelength dependence of the $\mathrm{THz}$ generation}

To further confirm that the $\mathrm{THz}$ emission from ITO is enhanced by the ENZ effect, we measure the pump wavelength dependence of the $\mathrm{THz}$ generation. In Fig. 3a, we plot the peak-to-peak amplitude of the $\mathrm{THz}$ signal as a function of the pump wavelength, with pump fluences of $0.78,3.12$, and $6.25 \mathrm{~mJ} / \mathrm{cm}^{2}$ in the transmission configuration. A redshift of the $\mathrm{THz}$ generation peak from 1340 to $1400 \mathrm{~nm}$ is observed as the pump fluence increases.

At the low pump fluence, the $\mathrm{THz}$ generation peak is blueshifted from the static $\lambda_{\mathrm{ENZ}}$ of the bulk ITO film measured through spectroscopic ellipsometry. This may be attributed to the nonlocal effect occurring in the ultrathin ITO film, as the electron doping concentration at the surface of the ITO film, where the $\mathrm{THz}$ wave is generated, may be higher than that in the bulk ITO film ${ }^{39,43}$. However, as the pump fluence increases, due to the photoinduced heating of conduction band electrons and the consequent time-dependent $\lambda_{\text {ENZ }}$ of ITO, the $\mathrm{THz}$ generation peak redshifts. As schematically shown in Fig. 3b, upon sub-bandgap photoexcitation, the conduction band electrons in ITO quickly thermalize into a hot Fermi distribution with a maximum electron temperature $T_{\mathrm{e}}$. Electrons then cool down and relax back to the conduction band minimum through energy exchange with phonons ${ }^{33,34}$. The $m_{\text {eff }}$ of ITO is a function of the electron distribution and $T_{\mathrm{e}}$ and is therefore time dependent, owing to the nonparabolicity of the conduction band. According to the Drude-Lorentz formula, an increase in $m_{\text {eff }}$ leads to a decrease in $\omega_{\mathrm{p}}$ and a redshift in the ITO's $\lambda_{\mathrm{ENZ}}$. The subpicosecond electron dynamics are comparable to the dwell time of the pump pulse inside the ITO cavity, which leads to the pump pulse interacting with a time-variant ITO cavity ${ }^{44}$. As a result, we observe the spectral redshift of the $\mathrm{THz}$ generation peak from the static $\lambda_{\text {ENZ }}$ of ITO. We develop a quantitative model based on the hot-electron dynamics of ITO to derive a static $\lambda_{\mathrm{ENZ}}$ of $1286 \mathrm{~nm}$ at the surface of the ITO film, which redshifts to $1340 \mathrm{~nm}$ under a pump fluence of $0.78 \mathrm{~mJ} / \mathrm{cm}^{2}$. Moreover, we calculate $\lambda_{\mathrm{ENZ}}$ as a function of the pump fluence (see Supplementary Note 3 for details), which explains the larger redshift when the ITO film is

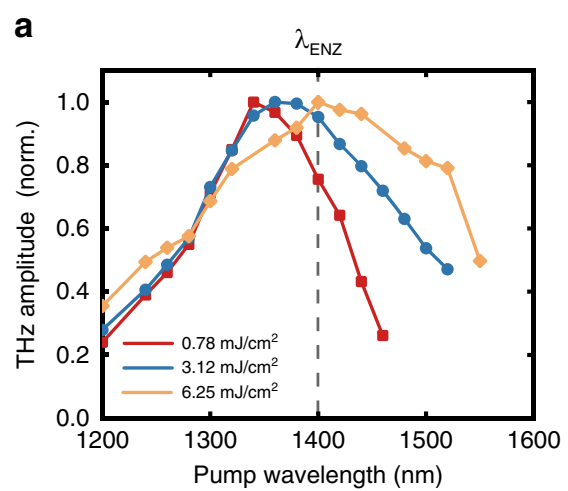

b
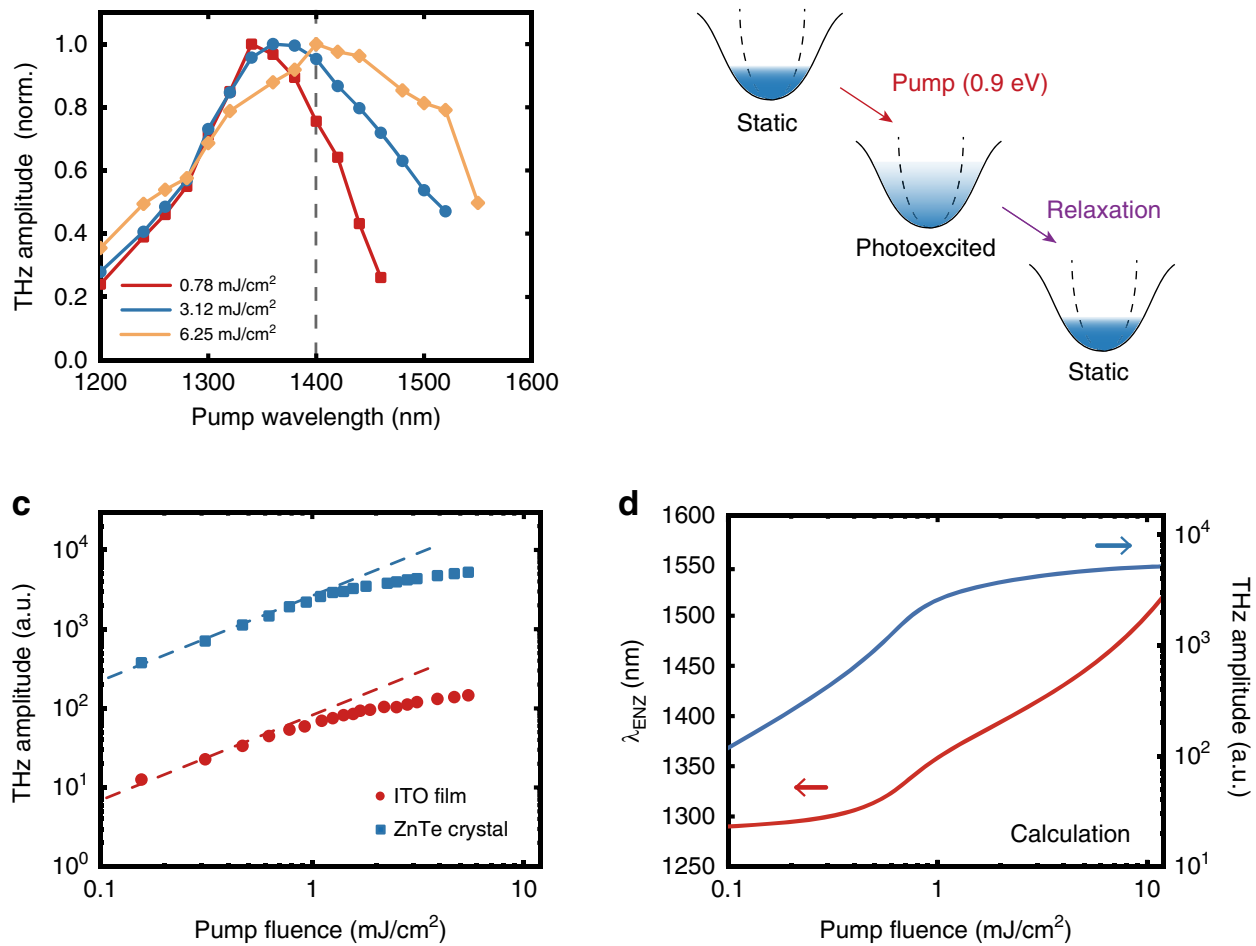

Fig. 3 Pump wavelength and pump fluence dependence of THz generation. a Measured peak-to-peak THz amplitude as a function of the pump wavelength with pump fluences of 0.78 (red), 3.12 (blue), and $6.25 \mathrm{~mJ} / \mathrm{cm}^{2}$ (yellow) in the transmission configuration. The dashed curve denotes the static ENZ wavelength of the bulk ITO film measured via spectroscopic ellipsometry. $\mathbf{b}$ Schematics of ultrafast electron dynamics in the ITO film, which consist of photoexcitation, hot-electron redistribution, and relaxation. The dashed line represents the parabolic conduction band approximation. c Measured peak-to-peak THz amplitude as a function of the pump fluence in the transmission configuration with a pump wavelength of $1350 \mathrm{~nm}$ for the ITO film (red) and $1 \mathrm{~mm}$-thick ZnTe crystal (blue). The dashed lines are from the linear fitting. $\mathbf{d}$ Calculated $\lambda_{\mathrm{ENZ}}$ of the ITO film (red) and THz amplitude (blue) as a function of the pump fluence in the transmission configuration 
excited with a higher pump fluence and is in good agreement with the literature studying the ultrafast dynamics of ENZ materials ${ }^{34,44}$.

\section{Pump fluence dependence of the $\mathrm{THz}$ generation}

To investigate the underlying physical mechanism of the $\mathrm{THz}$ generation in the ITO film, we also measure the peak-to-peak $\mathrm{THz}$ amplitude as a function of the pump fluence, as shown in Fig. 3c. Under low pump fluence, the $\mathrm{THz}$ amplitude scales linearly with the pump fluence, in agreement with the linear scaling law of the second-order nonlinear process of optical rectification. When the pump fluence exceeds $1.8 \mathrm{~mJ} / \mathrm{cm}^{2}$, the $\mathrm{THz}$ amplitude deviates from the linear scaling, which we again attribute to the time-dependent $\lambda_{\text {ENZ }}$ of ITO.

For optical rectification, the nonlinear $\mathrm{THz}$ polarization is $P_{\mathrm{THz}}=\chi_{\mathrm{eff}}^{(\mathrm{THz})} E_{0}^{2}$, where $E_{0}$ is the incident electric field amplitude. We define $\chi_{\text {eff }}^{(\mathrm{THz})}=\chi_{0}^{(\mathrm{THz})}\left(E_{\mathrm{ITO}} / E_{0}\right)^{2}$ as the effective second-order susceptibility of ITO, where $\chi_{0}^{(\mathrm{THz})}$ is a constant and $E_{\mathrm{ITO}}$ is the electric field amplitude in the ITO film. It is noteworthy that only the normal component of the electric field in ITO is enhanced under the ENZ condition. Under high pump fluence, the $\lambda_{\text {ENZ }}$ of ITO redshifts, leading to lower $E_{\mathrm{ITO}} / E_{0}$ and $\chi_{\text {eff }}^{(\mathrm{TH})}$. As a result, the $\mathrm{THz}$ generation efficiency declines gradually. We calculate the $\mathrm{THz}$ amplitude as a function of the pump fluence (see Supplementary Note 4 for details), which qualitatively agrees with the experimental results (Fig. 3d). The THz generation is reversible after repeated measurements with a pump fluence up to $10 \mathrm{~mJ} / \mathrm{cm}^{2}$, indicating no permanent damage to the ITO film.

In addition, we compare the $\mathrm{THz}$ generation efficiencies of the ITO film and a $1 \mathrm{~mm}$-thick ZnTe crystal under identical excitation conditions in the transmission configuration, although the phase-matching condition in the ZnTe crystal is not strictly satisfied at this pump wavelength (Fig. 3c). The thickness of the ITO film is five orders of magnitude less than that of the ZnTe crystal, yet the $\mathrm{THz}$ signal is less than two orders of magnitude weaker. The saturation effect in the $\mathrm{ZnTe}$ crystal can be attributed to a different origin of multiphoton absorption ${ }^{45,46}$.

\section{Bandwidth of the $\mathrm{THz}$ generation}

The generated $\mathrm{THz}$ spectra are obtained by the fast Fourier transform for various pump configurations and detection crystals, as shown in Fig. 4a. We observe a bandwidth of $\sim 3 \mathrm{THz}$ in all cases. In principle, the thickness of the ITO film is deep subwavelength with respect to the pump light such that it does not suffer from the $\mathrm{THz}$ generation bandwidth limitation imposed by the phase-matching condition. However, the pulse duration of the pump laser is approximately $100 \mathrm{fs}$, as measured by autocorrelation, corresponding to a bandwidth of only $4.4 \mathrm{THz}^{47}$. Moreover, the detection efficiency of the $\mathrm{ZnTe}$ crystal we use quickly declines beyond $3 \mathrm{THz}^{47}$. To verify that this is indeed an issue, we change the ZnTe crystal with a $1 \mathrm{~mm}$ thickness to another ZnTe crystal with a $0.1 \mathrm{~mm}$ thickness and observe an increase in the measured spectral bandwidth from 2.8 to $3.05 \mathrm{THz}$.

\section{Pump polarization and sample orientation dependence of the $\mathrm{THz}$ generation}

We further measure the peak-to-peak amplitude of $\mathrm{THz}$ signals versus the pump pulse polarization angle $\theta$, as shown schematically in Fig. 4b. We define $\theta$ to be $0^{\circ}$ and $90^{\circ}$ when the pump light is $s$-polarized and $p$-polarized, respectively. As shown in Fig. $4 \mathrm{c}$, the $p$-component of the $\mathrm{THz}$ amplitude well fits the $\sin ^{2}(\theta)$ function, consistent with the fact that the Ferrell-Berreman mode in ITO can only be excited with $p$-polarized light. Moreover, the $s$ component of the $\mathrm{THz}$ amplitude is $<10 \%$ of the $p$ component of the $\mathrm{THz}$ amplitude. Therefore, we can determine the normal component of the electric field $E_{\mathrm{z}}$ to be the main contributor to the $\mathrm{THz}$ generation, which is also in agreement with previous literature reporting second-harmonic generation in ITO films ${ }^{30}$. Moreover, we measure the $\mathrm{THz}$ amplitude as a function of the azimuthal angle $\varphi$ of the ITO film and find that the THz generation is not sensitive to the azimuthal angle, as expected from its amorphous structure (Fig. 4e). This can be another advantage of ITO film over other crystalline nonlinear crystals where the $\mathrm{THz}$ generation efficiency is highly dependent on the sample orientation.

\section{Discussion}

To summarize, we have observed broadband $\mathrm{THz}$ emission from commercially available ITO thin films in both the transmission and reflection configurations. We utilize the ENZ effect and the associated pump field enhancement in ITO to boost the $\mathrm{THz}$ generation efficiency. Due to the deep subwavelength thickness, the bandwidth of the $\mathrm{THz}$ emission from the ultrathin ITO film is over $3 \mathrm{THz}$, free from the phase-matching condition. Compared with optical metamaterials, the ITO film has a higher damage threshold ${ }^{19}$. Compared with bulk nonlinear crystals, the $\mathrm{THz}$ generation from the ITO film is not sensitive to the azimuthal angle. We attribute the origin of the $\mathrm{THz}$ emission to the surface optical rectification process. Its unique pump wavelength and fluence dependence may be attributed to the nonlocal effect and the unique hot-electron dynamics occurring in the ITO film. Currently, the $\mathrm{THz}$ generation efficiency from the ITO film is still much less than that of pulsed $\mathrm{THz}$ sources based on bulk nonlinear crystals, with a relatively low dynamic range on the order of hundreds, which hinders its imminent application. The $\mathrm{THz}$ generation efficiency may be further improved, e.g., by using high electron mobility ENZ materials such as $\mathrm{CdO}$, with a lower optical loss and a 


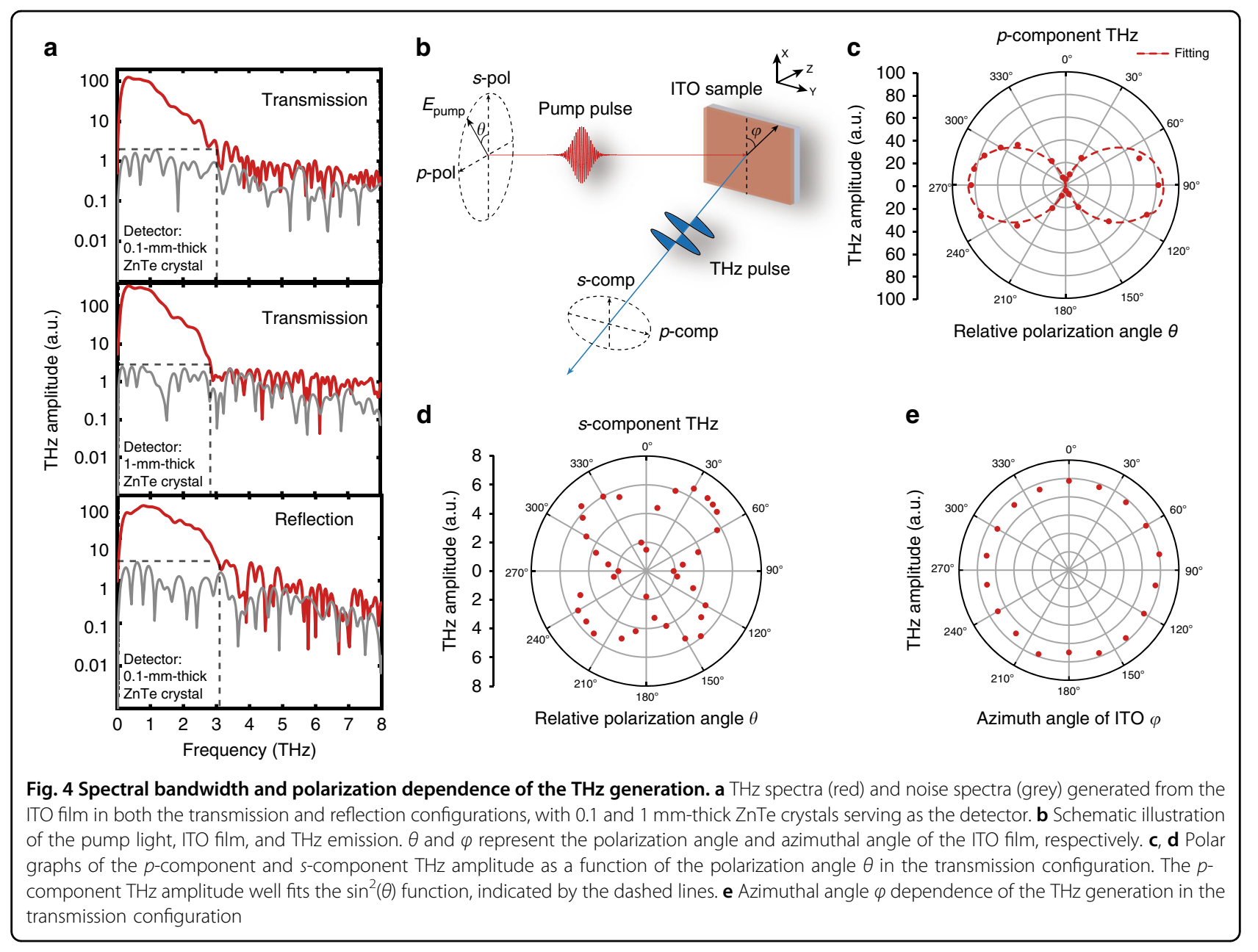

larger field enhancement ${ }^{44}$, and by coupling the ENZ material to optical metasurfaces ${ }^{48}$. Moreover, although the ITO film is currently pumped by a solid-state OPA, the $\lambda_{\text {ENZ }}$ of ITO can be tailored by controlling the doping concentration during film deposition and postdeposition annealing ${ }^{30,49}$. For example, an ITO film with a $\lambda_{\text {ENZ }}$ near $1550 \mathrm{~nm}$ can be directly pumped by a more compact fibrebased femtosecond laser with a lower system cost.

\section{Materials and methods}

\section{Optical measurement}

In the experiment, we use a Ti:sapphire amplifier system with a central wavelength of $800 \mathrm{~nm}$, a pulse duration of $35 \mathrm{fs}$, and a repetition rate of $1 \mathrm{kHz}$ as the pump source. The laser beam is divided into two beams by a beam splitter to generate and detect the $\mathrm{THz}$ signal. The main portion of the laser beam is used to pump an OPA to produce infrared pulses with a pulse duration of approximately $100 \mathrm{fs}$ and a wavelength tuneable from 1100 to $1600 \mathrm{~nm}$. The infrared pulse can be configured to excite the sample in either the transmission or reflection geometry. The incident angle of the pump beam is fixed at $40^{\circ}$ in the transmission configuration and $45^{\circ}$ in the reflection configuration. We have calculated the electric field enhancement factor as a function of wavelength and incident angle (see Supplementary Notes 5 and 6 for details). There is no significant difference in the field enhancement factor at $40^{\circ}$ and $45^{\circ}$ incidence angles. We did not use the larger incident angle due to the finite size of the ITO sample $\left(1 \mathrm{~cm}^{2}\right)$ and the space limitations for sample mounting in our current experimental setup. The sample is placed at the focus of a parabolic mirror, and the generated $\mathrm{THz}$ wave is focused on a $<110>$ cut $\mathrm{ZnTe}$ crystal by another parabolic mirror. The other portion of the laser beam from the amplifier system serves as the probe beam to implement electro-optical sampling of the $\mathrm{THz}$ signal through the use of the ZnTe crystal, a quarterwave plate (QWP), a Wollaston prism, and a pair of balanced photodiodes. It is noteworthy that the $\mathrm{ZnTe}$ crystal has the highest detection efficiency for the $p$ polarized $\mathrm{THz}$ signal. We can obtain the $\mathrm{THz}$ waveform by changing the time delay between the generated $\mathrm{THz}$ beam and the infrared probe beam. The polarization state of the pump beam is changed by rotating a half-wave plate and a 
polarizer. In the measurement, the time step is set to be $<0.02 \mathrm{ps}$ and the chopping frequency is set at $370 \mathrm{~Hz}$. The integration time of the lock-in amplifier is set at $300 \mathrm{~ms}$, with a sensitivity of $500 \mu \mathrm{V}$. The reported $\mathrm{THz}$ traces are the averaged results of over three measurements.

\section{Electro-optical sampling}

To detect the waveform of the generated $\mathrm{THz}$ signal, a linearly polarized probe pulse with a wavelength centred at $800 \mathrm{~nm}$ is sent into an electro-optical ZnTe crystal collinear with the THz pulse. Due to the Pockels effect of the $\mathrm{ZnTe}$ crystal, the birefringence induced by the $\mathrm{THz}$ electric field changes the polarization state of the probe pulse. When passing through a QWP, the probe pulse evolves into an elliptically polarized pulse. As it passes through a Wollaston prism, the probe pulse is split into two orthogonal components, which are then sent to a pair of balanced photodiodes. The balanced photodiodes measure the intensity difference $\Delta I$ between the two orthogonal components of the probe pulse, which is proportional to the amplitude of the $\mathrm{THz}$ signal $E_{\mathrm{THz}}$.

\section{Acknowledgements}

This work was supported by the National Key Research and Development Program of China (Grant number 2017YFA0701004) and the National Natural Science Foundation of China (Grant numbers 61975251, 61875150, 61735012, and 11774288).

\section{Author details \\ ${ }^{1}$ State Key Laboratory of Precision Measurement Technology and Instruments, Department of Precision Instrument, Tsinghua University, Beijing 100084, China. ${ }^{2}$ Center for THz Waves and College of Precision Instrument and Optoelectronics Engineering, Tianjin University, Tianjin 300072, China. ${ }^{3}$ Shaanxi Joint Lab of Graphene, International Collaborative Center on Photoelectric Technology and Nano Functional Materials, Institute of Photonics and Photon- Technology, Northwest University, Xi'an 710069, China. ${ }^{4}$ School of Electrical and Computer Engineering, Oklahoma State University, Stillwater, Oklahoma 74078, USA}

\section{Author contributions}

Y.Y. and J.H. conceived the idea. W.J., M.L., Y.L., X.F., Q.W., X.Z., Y.N., and Y.H. performed the experiments. F.H. and W.J. developed the theory. All authors discussed the results and contributed to writing the manuscript. Y.Y. and J.H. supervised the project.

\section{Conflict of interest}

The authors declare that they have no conflict of interest.

Supplementary information is available for this paper at https://doi.org/ 10.1038/s41377-020-00452-y.

Received: 3 July 2020 Revised: 3 December 2020 Accepted: 7 December 2020

Published online: 07 January 2021

\footnotetext{
References

1. Siegel, P. H. Terahertz technology. IEEE Trans. Microw. Theory Tech. 50, 910-928 (2002).

2. Tonouchi, M. Cutting-edge terahertz technology. Nat. Photonics 1, 97-105 (2007).
}

3. Ferguson, B. \& Zhang, X. C. Materials for terahertz science and technology. Nat. Mater. 1, 26-33 (2002).

4. Palka, N., Szala, M. \& Czerwinska, E. Characterization of prospective explosive materials using terahertz time-domain spectroscopy. Appl. Opt. 55, 4575-4583 (2016).

5. Krimi, S. et al. Highly accurate thickness measurement of multi-layered automotive paints using terahertz technology. Appl. Phys. Lett. 109, 021105 (2016).

6. Wade, C. G. et al. Real-time near-field terahertz imaging with atomic optical fluorescence. Nat. Photonics 11, 40-43 (2017).

7. Stantchev, R. I. et al. Noninvasive, near-field terahertz imaging of hidden objects using a single-pixel detector. Sci. Adv. 2, e1600190 (2016).

8. Liu, J. L. et al. Broadband terahertz wave remote sensing using coherent manipulation of fluorescence from asymmetrically ionized gases. Nat. Photonics 4, 627-631 (2010).

9. $\mathrm{Xu}, \mathrm{W}$. D. et al. Gold nanoparticle-based terahertz metamaterial sensors: mechanisms and applications. ACS Photonics 3, 2308-2314 (2016).

10. Song, H. J. \& Nagatsuma, T. Present and future of terahertz communications. IEEE Trans. Terahertz Sci. Technol. 1, 256-263 (2011).

11. Nagatsuma, T., Ducournau, G. \& Renaud, C. C. Advances in terahertz communications accelerated by photonics. Nat. Photonics 10, 371-379 (2016).

12. Dai, J. M., Liu, J. L. \& Zhang, X. C. Terahertz wave air photonics: terahertz wave generation and detection with laser-induced gas plasma. IEEE J. Sel. Top. Quantum Electron. 17, 183-190 (2011).

13. Zhao, J. Y. et al. Strong spatial confinement of terahertz wave inside femtosecond laser filament. ACS Photonics 3, 2338-2343 (2016).

14. Jin, Q. et al. Observation of broadband terahertz wave generation from liquid water. Appl. Phys. Lett. 111, 071103 (2017).

15. Blanchard, F. et al. Generation of $1.5 \mu$ single-cycle terahertz pulses by optical rectification from a large aperture ZnTe crystal. Opt. Express 15, 13212-13220 (2007).

16. Aoki, K., Savolainen, J. \& Havenith, M. Broadband terahertz pulse generation by optical rectification in GaP crystals. Appl. Phys. Lett. 110, 201103 (2017).

17. $\mathrm{Wu}, \mathrm{X}$. J. et al. Terahertz generation in lithium niobate driven by Ti:sapphire laser pulses and its limitations. Opt. Lett. 39, 5403-5406 (2014).

18. Luo, L. et al. Broadband terahertz generation from metamaterials. Nat. Commun. 5, 3055 (2014)

19. Fang, M. et al. Nonlinearity in the dark: broadband terahertz generation with extremely high efficiency. Phys. Rev. Lett. 122, 027401 (2019).

20. Bahk, Y. M. et al. Plasmon enhanced terahertz emission from single layer graphene. ACS Nano 8, 9089-9096 (2014).

21. Zhang, L. H. et al. Polarized THz emission from in-plane dipoles in monolayer tungsten disulfide by linear and circular optical rectification. Adv. Opt. Mater. 7, 1801314 (2019).

22. Arlauskas, A. et al. Strong terahertz emission and its origin from catalyst-free InAs nanowire arrays. Nano Lett. 14, 1508-1514 (2014).

23. Arlauskas, A. \& Krotkus, A. THz excitation spectra of AlllBV semiconductors. Semiconductor Sci. Technol. 27, 115015 (2012).

24. Campione, S., Brener, I. \& Marquier, F. Theory of epsilon-near-zero modes in ultrathin films. Phys. Rev. B 91, 121408 (2015).

25. Vassant, S. et al. Berreman mode and epsilon near zero mode. Opt. Express 20, 23971-23977 (2012).

26. Alam, M. Z., De Leon, I. \& Boyd, R. W. Large optical nonlinearity of indium tin oxide in its epsilon-near-zero region. Science 352, 795-797 (2016).

27. Reshef, O. et al. Nonlinear optical effects in epsilon-near-zero media. Nat. Rev Mater. 4, 535-551 (2019)

28. Kinsey, N. et al. Near-zero-index materials for photonics. Nat. Rev. Mater. 4 742-760 (2019)

29. Niu, X. X. et al. Epsilon-near-zero photonics: a new platform for integrated devices. Adv. Opt. Mater. 6, 1701292 (2018).

30. Capretti, A. et al. Comparative study of second-harmonic generation from epsilon-near-zero indium tin oxide and titanium nitride nanolayers excited in the near-infrared spectral range. ACS Photonics 2, 1584-1591 (2015).

31. Capretti, A. et al. Enhanced third-harmonic generation in Si-compatible epsilon-near-zero indium tin oxide nanolayers. Opt. Lett. 40, 1500-1503 (2015).

32. Luk, T. S. et al. Enhanced third harmonic generation from the epsilon-nearzero modes of ultrathin films. Appl. Phys. Lett. 106, 151103 (2015).

33. Yang, Y. M. et al. Femtosecond optical polarization switching using a cadmium oxide-based perfect absorber. Nat. Photonics 11, 390-395 (2017).

34. Guo, P. J. et al. Ultrafast switching of tunable infrared plasmons in indium tin oxide nanorod arrays with large absolute amplitude. Nat. Photonics 10 267-273 (2016) 
35. Anopchenko, A. et al. Field-effect tunable and broadband epsilon-near-zero perfect absorbers with deep subwavelength thickness. ACS Photonics $\mathbf{5}$ 2631-2637 (2018).

36. Khurgin, J. B. et al. Adiabatic frequency shifting in epsilon-near-zero materials: the role of group velocity. Optica 7, 226-231 (2020).

37. Noginov, M. A. et al. Transparent conductive oxides: plasmonic materials for telecom wavelengths. Appl. Phys. Lett. 99, 021101 (2011).

38. Caldwell, J. D. et al. Low-loss, infrared and terahertz nanophotonics using surface phonon polaritons. Nanophotonics 4, 44-68 (2015).

39. Rodriguez-Suné, L. et al. Study of second and third harmonic generation from an indium tin oxide nanolayer: influence of nonlocal effects and hot electrons. APL Photonics 5, 010801 (2020).

40. Newman, W. D. et al. Ferrell-Berreman modes in plasmonic epsilon-near-zero media. ACS Photonics 2, 2-7 (2015).

41. Scalora, M. et al. Harmonic generation from metal-oxide and metal-metal boundaries. Phys. Rev. A 98, 023837 (2018).
42. Dass, C. K . et al. Gap-plasmon-enhanced second-harmonic generation in epsilon-near-zero nanolayers. ACS Photonics 7, 174-179 (2020).

43. Scalora, M. et al. Electrodynamics of conductive oxides: intensity-dependent anisotropy, reconstruction of the effective dielectric constant, and harmonic generation. Phys. Rev. A 101, 053828 (2020).

44. Yang, Y. M. et al. High-harmonic generation from an epsilon-near-zero material. Nat. Phys. 15, 1022-1026 (2019).

45. Löffler, T. et al. Large-area electro-optic ZnTe terahertz emitters. Opt. Express 13, 5353-5362 (2005).

46. Hoffmann, M. C. et al. Efficient terahertz generation by optical rectification at 1035 nm. Opt. Express 15, 11706-11713 (2007).

47. Lee, Y. S. Principles of Terahertz Science and Technology (Springer, Boston, 2009).

48. Alam, M. Z. et al. Large optical nonlinearity of nanoantennas coupled to an epsilon-near-zero material. Nat. Photonics 12, 79-83 (2018).

49. Naik, G. V., Kim, J. \& Boltasseva, A. Oxides and nitrides as alternative plasmonic materials in the optical range [Invited]. Opt. Mater. Express 1, 1090-1099 (2011). 